\title{
Copper-Catalyzed Amination in the Synthesis of Polyoxadiamine Derivatives of Aza- and Diazacrown Ethers
}

\author{
Alexei A. Yakushev, ${ }^{\mathrm{a}, \mathrm{c}}$ Alexei D. Averin, ${ }^{\mathrm{a}, \mathrm{b} @ ~ M a x i m ~ V . ~ A n o k h i n, ~}{ }^{\mathrm{b}}$

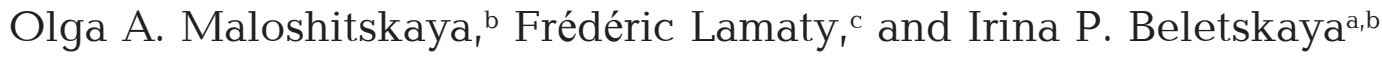 \\ ${ }^{a}$ A.N. Frumkin Institute of Physical Chemistry and Electrochemistry, 119991 Moscow, Russia \\ ${ }^{\mathrm{b}}$ Lomonosov Moscow State University, Department of Chemistry, 119991 Moscow, Russia \\ 'Institut des Biomolécules Max Mousseron (IBMM) UMR 5247, Université Montpellier 2, 34095 Montpellier Cedex, France \\ ${ }^{\circledR}$ Corresponding author E-mail: alexaveron@yandex.ru
}

\begin{abstract}
$\mathrm{Cu}(\mathrm{I})$-Catalyzed amination of $\mathrm{N}$-(iodobenzyl) substituted azacrown ethers with propane-1,3-diamine and several polyoxadiamines was investigated. In the case of diamine excess and the use of CuI/l-proline catalytic system in EtCN the formation of the compounds with one azacrown and one diamine moieties was observed. When taking the excess of $N$-(iodobenzyl) derivative of azacrown ether and CuI/2-(isobutyryl)cyclohexanone catalytic system in DMF, bis(azacrown) substituted oxadiamine could be obtained. Amination of N,N'-di(iodobenzyl) substituted diazacrown ether was studied under the same conditions. The comparison of Pd(0)-with $\mathrm{Cu}(\mathrm{I})$-mediated amination reactions was done using $N$-(bromobenzyl) substituted azacrown ethers.
\end{abstract}

Keywords: Catalysis by $\mathrm{Cu}(\mathrm{I})$ complexes, amination, azacrown ethers, diamines.

\section{Медь-катализируемое аминирование в синтезе полиоксадиаминовых производных аза- и диазакраун-эфиров}

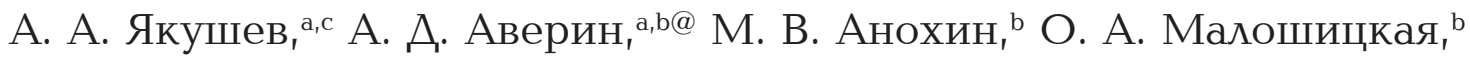 \\ Ф. Ламати, ${ }^{c}$ И. П. Белецкая ${ }^{\mathrm{a}, \mathrm{b}}$ \\ а Институт физической химии и электрохимии им. А.Н. Фрумкина, 119991 Москва, Россия \\ ${ }^{\mathrm{b}}$ Московский государственный университет им. М.В. Ломоносова, Химический факультет, 119991 Москва, Россия \\ 'Institut des Biomolécules Max Mousseron (IBMM) UMR 5247, Университет Монпелье 2, 34095 Монпелье Седекс, \\ Франичия \\ @E-mail: alexaveron@yandex.ru
}

\begin{abstract}
Изучено Си(I)-катализируемое аминирование $N$-(иодбензил) замещеенных азакраун-эфиров пропан-1,3-диамином и некоторыми полиоксадиаминами. При использовании избытка диаминов и каталитической системь CuI/l-пролин в EtCN наблюдалось образование соединений, содержащих один азакраун-эфирный и диаминовый фрагментыл. При использовании избытка $N$-(иодбензил) производных азакраун-эфиров и каталитической системы СиІ/2-(изобутирил)циклогексанон в ДМФА могут быть получены бис(азакраун) замещенные оксадиамины. Аминирование N,N’-ди(иодбензил) замещзенного дизакраун-эфира исследовано в тех же условиях. Проведено сравнение Pd(0)- и Cu(I)-катализируемого аминирования с использованием $N$-(бромбензил) замещенных азакраун-эфиров.
\end{abstract}

Ключевые слова: $\mathrm{Cu}(\mathrm{I})-$ Катализ, аминирование, азакраун-эфиры, диамины. 


\section{Introduction}

Oxygen- and nitrogen-containing macrocyclic compounds attract researchers' attention due to their unique properties of efficient and selective binding of metal cations, inorganic anions and small polar organic molecules. Introduction of additional donor atoms in the exocyclic substituents of such macrocycles may increase selectivity, binding constants and thus is widely used in coordination and supramolecular chemistry. One of the most important and renowned compounds of such type is $N, N^{\prime}, N$,', $N$ '”-tetrasubstituted 1,4,7,10tetraazacyclododecane (DOTA) bearing four carboxylic groups. It has been widely used in the coordination studies with various metal cations including rare earth metals and lanthanides. ${ }^{[1-4]}$ Well-studied are other tetrasubstituted tetraazamacrocycles like cyclen and cyclam $(1,4,8,11$-tetraazacyclotetradecane) with phosphonate, ${ }^{[5-10]}$ amide, ${ }^{[1]}$ nitrile, ${ }^{[12]}$ primary and tertiary amino groups. ${ }^{[13,14]}$ In many cases additional donor atoms are present in C-substituted macrocycles like cyclam ${ }^{[15,16]}$ or crown ethers. ${ }^{[17]}$ Special interest is paid to substituents bearing several donor atoms (podands), among them oligoethylene oxides are very attractive due to an easy introduction of such podands into macrocycles, possibility to vary the number of donor atoms and their non-ionogenic character. Aza-crown ethers are usually modified with these podands at the nitrogen atom, ${ }^{[18-20]}$ while crown ethers may contain these substituents only at carbon atoms. ${ }^{[21-23]}$ We have developed a simple and efficient approach to macrobicycles and trismacrocycles compounds using $\operatorname{Pd}(0)$-catalyzed macrocyclization of $N, N^{\prime}$-di(bromobenzyl) substituted diazacrown ethers with polyamines and polyoxadiamines, ${ }^{[24,25]}$ also we carried out the synthesis of various bismacrocycles based on $N$-benzyl substituted azacrown ethers. ${ }^{[26]}$ Recently we have started thourough investigation of $\mathrm{Cu}(\mathrm{I})$-catalyzed arylation and heteroarylation of polyamines and polyoxadiamines ${ }^{[27-29]}$ as well as $\mathrm{Cu}(\mathrm{I})$-mediated amination of steroids. ${ }^{[30]}$ In this connection, the aim of the present research is to apply $\mathrm{Cu}(\mathrm{I})$ catalyzed amination to the synthesis of polyoxadiamine derivatives of aza- and diazacrown ethers and to compare it with the approach which exploits $\operatorname{Pd}(0)$ catalysis.

\section{Experimental}

NMR spectra were registered using Bruker Avance 400 spectrometer, MALDI-TOF spectra were obtained with Bruker Autoflex II spectrometer using 1,8,9-trihydroxyanthracene as matrix and PEGs as internal standards. Propane-1,3-diamine, dioxaand trioxadiamines, aza- and diazacrown ethers, 1-(bromomethyl)3-bromobenzene, l-proline, 2-isobutyrylcyclohexanone, BINAP, cesium carbonate, potassium carbonate, sodium tert-butoxide, $\mathrm{CuI}$ were purchased from Aldrich and used without further purification, $\operatorname{Pd}(\mathrm{dba})_{2}$ was synthesized according to the method described,,${ }^{[31]}$ 1-(bromomethyl)-3-iodobenzene was obtained from commercially available $m$-iodotoluene by a standard bromination with NBS in $\mathrm{CCl}_{4}$. Dioxane was distilled over $\mathrm{NaOH}$ followed by the distillation over sodium under argon, acetonitrile was distilled over $\mathrm{CaH}_{2}, \mathrm{DMF}$, EtCN, dichloromethane and methanol were used freshly distilled.

Typical procedure for the synthesis of aza- and diazacrown derivatives 1-4, 9. A flask equipped with a condenser and magnetic stirrer was charged with corresponding azacrown ether (1 mmol), MeCN (3 ml), 1-(bromomethyl)-3-bromobenzene or 1-(bromomethyl)-3-iodobenzene $(1 \mathrm{mmol})$, potassium carbonate
$(2.5 \mathrm{mmol})$ and stirred under reflux for $15 \mathrm{~h}$. In the case of compound 9 diaza-18-crown-6 ether $(1 \mathrm{mmol})$ was reacted with 1-(bromomethyl)-3-iodobenzene $(2 \mathrm{mmol})$ in the presence of potassium carbonate $(4 \mathrm{mmol})$. After cooling the reaction mixture down to room temperature the reaction mixture was diluted with $\mathrm{CH}_{2} \mathrm{Cl}_{2}(10 \mathrm{ml})$, the solution was filtered, evaporated in vacuo, dissolved in $\mathrm{CH}_{2} \mathrm{Cl}_{2}(10 \mathrm{ml})$, washed with an equal volume of water and dried over molecular sieves. After evaporating the solvent, target $N$-halogenobenzyl derivatives of aza- and diazacrown ethers were obtained as viscous yellowish oils.

13-(3-Bromobenzyl)-1,4,7,10-tetraoxa-13-azacyclopentadecane (1). Obtained from 1-aza-15-crown-5 (2 mmol, $438 \mathrm{mg})$ and 3-bromobenzyl bromide ( $2 \mathrm{mmol}, 500 \mathrm{mg}$ ) in the presence of $\mathrm{K}_{2} \mathrm{CO}_{3}(5 \mathrm{mmol}, 695 \mathrm{mg})$ in $7 \mathrm{ml} \mathrm{MeCN}$. Yield $728 \mathrm{mg}(94 \%)$. (MALDI-TOF) found: 388.1167. $\mathrm{C}_{17} \mathrm{H}_{27} \mathrm{BrNO}_{4}$ requires 388.1123 $[\mathrm{M}+\mathrm{H}]^{+} .{ }^{1} \mathrm{H} \mathrm{NMR}\left(\mathrm{CDCl}_{3}, 298 \mathrm{~K}\right) \delta_{\mathrm{H}} \mathrm{ppm}: 2.76\left(4 \mathrm{H}, \mathrm{t},{ }^{3} J=5.8 \mathrm{~Hz}\right)$, 3.60-3.64 (10H, m), 3.65-3.69 $(8 \mathrm{H}, \mathrm{m}), 7.13\left(1 \mathrm{H}, \mathrm{t},{ }^{3} J=7.8 \mathrm{~Hz}\right)$, $7.24\left(1 \mathrm{H}, \mathrm{d},{ }^{3} J=7.8 \mathrm{~Hz}\right), 7.33\left(1 \mathrm{H}, \mathrm{d},{ }^{3} J=7.8 \mathrm{~Hz}\right), 7.51(1 \mathrm{H}, \mathrm{s})$. ${ }^{13} \mathrm{C} \mathrm{NMR}\left(\mathrm{CDCl}_{3}, 298 \mathrm{~K}\right) \delta_{\mathrm{c}} \mathrm{ppm}: 54.2(2 \mathrm{C}), 60.1(1 \mathrm{C}), 69.9(2 \mathrm{C})$, 70.2 (2C), 70.5 (2C), 70.9 (2C), 122.4 (1C), 127.3 (1C), 129.7 (1C), 129.9 (1C), $131.6(1 \mathrm{C}), 142.3(1 \mathrm{C})$.

16-(3-Bromobenzyl)-1,4,7,10,13-pentaoxa-16-azacyclooctadecane (2). Obtained from 1-aza-18-crown-6 (1 mmol, $263 \mathrm{mg})$ and 3-bromobenzyl bromide ( $1 \mathrm{mmol}, 250 \mathrm{mg}$ ) in the presence of $\mathrm{K}_{2} \mathrm{CO}_{3}(2.5 \mathrm{mmol}, 348 \mathrm{mg}$ ) in $4 \mathrm{ml} \mathrm{MeCN}$. Yield $415 \mathrm{mg}(96 \%)$. (MALDI-TOF) found: 432.1425. $\mathrm{C}_{19} \mathrm{H}_{31} \mathrm{BrNO}_{5}$ requires 432.1386 $[\mathrm{M}+\mathrm{H}]^{+} .{ }^{1} \mathrm{H} \mathrm{NMR}\left(\mathrm{CDCl}_{3}, 298 \mathrm{~K}\right) \delta_{\mathrm{H}} \mathrm{ppm}: 2.76\left(4 \mathrm{H}, \mathrm{t},{ }^{3} J=5.3 \mathrm{~Hz}\right)$, 3.57-3.62 (8H, m), 3.63-3.69 $(14 \mathrm{H}, \mathrm{m}), 7.13\left(1 \mathrm{H}, \mathrm{t},{ }^{3} J=7.7 \mathrm{~Hz}\right)$, $7.24\left(1 \mathrm{H}, \mathrm{d},{ }^{3} \mathrm{~J}=8.0 \mathrm{~Hz}\right), 7.32\left(1 \mathrm{H}, \mathrm{d},{ }^{3} J=7.6 \mathrm{~Hz}\right), 7.50(1 \mathrm{H}, \mathrm{s})$. ${ }^{13} \mathrm{C} \mathrm{NMR}\left(\mathrm{CDCl}_{3}, 298 \mathrm{~K}\right) \delta_{\mathrm{c}} \mathrm{ppm}$ : $53.9(2 \mathrm{C}), 59.5(1 \mathrm{C}), 69.9(2 \mathrm{C})$, 70.4 (2C), 70.8 (4C), 70.9 (2C), 122.4 (1C), 127.3 (1C), 129.7 (1C), 129.9 (1C), $131.6(1 \mathrm{C}), 142.5(1 \mathrm{C})$.

13-(3-Iodobenzyl)-1,4,7,10-tetraoxa-13-azacyclopentadecane (3). Obtained from 1-aza-15-crown-5 (4 mmol, $876 \mathrm{mg})$ and 3-iodobenzyl bromide (4 mmol, $1188 \mathrm{mg})$ in the presence of $\mathrm{K}_{2} \mathrm{CO}_{3}(8 \mathrm{mmol}, 1104 \mathrm{mg}$ ) in $12 \mathrm{ml} \mathrm{MeCN}$. Yield $1.600 \mathrm{~g}(92 \%)$. (MALDI-TOF) found: 436.0932. $\mathrm{C}_{17} \mathrm{H}_{27} \mathrm{INO}_{4}$ requires 436.0985 $[\mathrm{M}+\mathrm{H}]^{+} .{ }^{1} \mathrm{H} \mathrm{NMR}\left(\mathrm{CDCl}_{3}, 298 \mathrm{~K}\right) \delta_{\mathrm{H}} \mathrm{ppm}: 2.70\left(4 \mathrm{H}, \mathrm{t},{ }^{3} \mathrm{~J}=5.9 \mathrm{~Hz}\right)$, 3.54-3.59 (10H, m), 3.60-3.64 (8H, m), $6.96\left(1 \mathrm{H}, \mathrm{t},{ }^{3} J=7.7 \mathrm{~Hz}\right)$, $7.23\left(1 \mathrm{H}, \mathrm{d},{ }^{3} J=7.6 \mathrm{~Hz}\right), 7.49\left(1 \mathrm{H}, \mathrm{d},{ }^{3} J=7.8 \mathrm{~Hz}\right), 7.65(1 \mathrm{H}, \mathrm{s})$.

16-(3-Iodobenzyl)-1,4, 7, 10,13-pentaoxa-16-azacyclooctadecane (4). Obtained from 1-aza-18-crown-6 (1 mmol, $263 \mathrm{mg})$ and 3-iodobenzyl bromide ( $1 \mathrm{mmol}, 297 \mathrm{mg}$ ) in the presence of $\mathrm{K}_{2} \mathrm{CO}_{3}(2.5 \mathrm{mmol}, 348 \mathrm{mg})$ in $4 \mathrm{ml} \mathrm{MeCN}$. Yield $445 \mathrm{mg}(93 \%)$. (MALDI-TOF) found: 480.1289. $\mathrm{C}_{19} \mathrm{H}_{31} \mathrm{INO}_{5}$ requires 480.1247 $[\mathrm{M}+\mathrm{H}]^{+} .{ }^{1} \mathrm{H} \mathrm{NMR}\left(\mathrm{CDCl}_{3}, 298 \mathrm{~K}\right) \delta_{\mathrm{H}} \mathrm{ppm}: 2.76\left(4 \mathrm{H}, \mathrm{t},{ }^{3} \mathrm{~J}=5.7 \mathrm{~Hz}\right)$, 3.59-3.64 (10H, m), 3.64-3.70 (12H, m), $7.01\left(1 \mathrm{H}, \mathrm{t},{ }^{3} J=7.7 \mathrm{~Hz}\right)$, $7.29\left(1 \mathrm{H}, \mathrm{d},{ }^{3} J=7.7 \mathrm{~Hz}\right), 7.54\left(1 \mathrm{H}, \mathrm{d},{ }^{3} J=7.8 \mathrm{~Hz}\right), 7.70(1 \mathrm{H}, \mathrm{s})$. ${ }^{13} \mathrm{C} \mathrm{NMR}\left(\mathrm{CDCl}_{3}, 298 \mathrm{~K}\right) \delta_{\mathrm{c}} \mathrm{ppm}: 53.9(2 \mathrm{C}), 59.4(1 \mathrm{C}), 69.9(2 \mathrm{C})$, 70.4 (2C), 70.8 (4C), 70.9 (2C), 104.5 (1C), 128.0 (1C), 129.9 (1C), 135.9 (1C), $137.6(1 \mathrm{C}), 142.6(1 \mathrm{C})$.

7,16-Bis(3-iodobenzyl)-1,4,10,13-tetraoxa-7,16-diazacyclooctadecane (9). Obtained from diaza-18-crown-6 (4 mmol, $1.060 \mathrm{~g})$ and 3-iodobenzyl bromide ( $8 \mathrm{mmol}, 2.376 \mathrm{~g})$ in the presence of $\mathrm{Na}_{2} \mathrm{CO}_{3}(16 \mathrm{mmol}, 1.696 \mathrm{~g}$ ) in $15 \mathrm{ml} \mathrm{MeCN}$. Yield $2.498 \mathrm{~g}$ (90\%). (MALDI-TOF) found: 695.0802. $\mathrm{C}_{26} \mathrm{H}_{37} \mathrm{I}_{2} \mathrm{~N}_{2} \mathrm{O}_{4}$ requires $695.0843[\mathrm{M}+\mathrm{H}]^{+} .{ }^{1} \mathrm{H} \mathrm{NMR}\left(\mathrm{CDCl}_{3}, 298 \mathrm{~K}\right) \delta_{\mathrm{H}} \mathrm{ppm}: 2.76(8 \mathrm{H}, \mathrm{t}$, $\left.{ }^{3} J=5.3 \mathrm{~Hz}\right), 3.52-3.65(20 \mathrm{H}, \mathrm{m}), 6.98\left(2 \mathrm{H}, \mathrm{t},{ }^{3} J=7.7 \mathrm{~Hz}\right), 7.26(2 \mathrm{H}, \mathrm{d}$, $\left.{ }^{3} J=7.7 \mathrm{~Hz}\right), 7.51\left(2 \mathrm{H}, \mathrm{d},{ }^{3} J=7.7 \mathrm{~Hz}\right), 7.67(2 \mathrm{H}, \mathrm{s})$.

Typical procedure for the $\mathrm{Cu}(\mathrm{I})$-catalyzed amination of compounds 3, 4, 9. A two-necked flask equipped with a condenser and magnetic stirrer was flushed with argon, charged with corresponding azacrown ether derivative 3, 4, 9 ( $0.5 \mathrm{mmol}$ for the monoamination or diamination or $1 \mathrm{mmol}$ for the diarylation), $\mathrm{CuI}$ (10 $\mathrm{mol} \%$ for the monoamiantion or $20 \mathrm{~mol} \%$ for the diamination and diarylation), $l$-proline or 2-isobutyrylcyclohexanone (20 $\mathrm{mol} \%$ for the monoamination or $40 \mathrm{~mol} \%$ for the diamination and diarylation), EtCN or DMF $(1 \mathrm{ml})$, corresponding diamine/ 
oxadiamine ( $1 \mathrm{mmol}$ for the monoamination, $2 \mathrm{mmol}$ for the diamination, or $0.5 \mathrm{mmol}$ for the diarylation), cesium carbonate (1.5 equiv. for each $\mathrm{NH}_{2}$ group participating in the arylation). The reaction mixture was stirred under reflux $(\mathrm{EtCN})$ or at $140{ }^{\circ} \mathrm{C}$ (DMF) for $24 \mathrm{~h}$. After cooling the reaction mixture down to room temperature the reaction mixture was diluted with $\mathrm{CH}_{2} \mathrm{Cl}_{2}(10 \mathrm{ml})$, the solution was filtered, evaporated in vacuo, dissolved in $\mathrm{CH}_{2} \mathrm{Cl}_{2}$ $(10 \mathrm{ml})$, and additional precipitate was separated. After evaporating the solvent, the residue was chromatographed on silica gel using a sequence of eluents: $\mathrm{CH}_{2} \mathrm{Cl}_{2}, \mathrm{CH}_{2} \mathrm{Cl}_{2} / \mathrm{MeOH}$ (50:1-3:1), $\mathrm{CH}_{2} \mathrm{Cl}_{2} /$ $\mathrm{MeOH} / \mathrm{NH}_{3}$ aq (100:20:1-10:4:1).

Typical procedure for the Pd(0)-catalyzed synthesis of compounds $7 \boldsymbol{a}, \boldsymbol{b}$. A two-necked flask equipped with a condenser and magnetic stirrer was flushed with argon, charged with corresponding azacrown ether derivative 1 or $2(0.5 \mathrm{mmol}), \mathrm{Pd}(\mathrm{dba})_{2}(4 \mathrm{~mol} \%$, $12 \mathrm{mg})$, BINAP (4.5 mol\%, $14 \mathrm{mg})$, absolute dioxane $(10-20 \mathrm{ml})$, propane-1,3-diamine (1.5 or $2 \mathrm{mmol}, 111$ or $148 \mathrm{mg}$ ) was added followed by sodium tert-butoxide $(0.75 \mathrm{mmol}, 72 \mathrm{mg})$. The reaction mixture was refluxed for $8 \mathrm{~h}$, after cooling the reaction mixture down to room temperature the reaction mixture was diluted with $\mathrm{CH}_{2} \mathrm{Cl}_{2}(10 \mathrm{ml})$, the solution was filtered, evaporated in vacuo, dissolved in $\mathrm{CH}_{2} \mathrm{Cl}_{2}(10 \mathrm{ml})$, and additional precipitate was separated. After evaporating the solvent, the residue was chromatographed on silica gel using a sequence of eluents: $\mathrm{CH}_{2} \mathrm{Cl}_{2}, \mathrm{CH}_{2} \mathrm{Cl}_{2} / \mathrm{MeOH}$ (50:1-3:1), $\mathrm{CH}_{2} \mathrm{Cl}_{2} / \mathrm{MeOH} / \mathrm{NH}_{3}$ aq $(100: 20: 1-10: 4: 1)$ to produce target compounds as viscous yellowish oils or glassy solids.

$N^{1}$-(3-((1,4, 7,10-Tetraoxa-13-azacyclopentadecan-13-yl)-methyl)phenyl)propane-1,3-diamine (6a). Obtained from compound $\mathbf{1}$ (1 $\mathrm{mmol}, 388 \mathrm{mg}$ ), propane-1,3-diamine (5a) (3 mmol, $222 \mathrm{mg})$, in the presence of $\mathrm{Pd}(\mathrm{dba})_{2}(4 \mathrm{~mol} \%, 24 \mathrm{mg})$, BINAP (4.5 mol\%, $28 \mathrm{mg}), t \mathrm{BuONa}(1.5 \mathrm{mmol}, 144 \mathrm{mg})$ in $20 \mathrm{ml}$ dioxane. Eluent: $\mathrm{CH}_{2} \mathrm{Cl}_{2} / \mathrm{MeOH} / \mathrm{NH}_{3}$ aq (100:25:5-100:35:6). Yield $221 \mathrm{mg}$ (58\%). (MALDI-TOF) found: 382.2670. $\mathrm{C}_{20} \mathrm{H}_{36} \mathrm{~N}_{3} \mathrm{O}_{4}$ requires 382.2706 $[\mathrm{M}+\mathrm{H}]^{+} .{ }^{1} \mathrm{H}$ NMR $\left(\mathrm{CDCl}_{3}, 298 \mathrm{~K}\right) \delta_{\mathrm{H}} \mathrm{ppm}: 1.75(2 \mathrm{H}$, quintet, $\left.{ }^{3} J=6.6 \mathrm{~Hz}\right), 2.73\left(4 \mathrm{H}, \mathrm{t},{ }^{3} \mathrm{~J}=5.7 \mathrm{~Hz}\right), 2.79\left(2 \mathrm{H}\right.$, br. $\left.\mathrm{t},{ }^{3} J_{\mathrm{obs}}=6.2 \mathrm{~Hz}\right)$, $3.14\left(2 \mathrm{H}, \mathrm{t},{ }^{3} \mathrm{~J}=6.6 \mathrm{~Hz}\right), 3.56(2 \mathrm{H}, \mathrm{s}), 3.57-3.65(16 \mathrm{H}, \mathrm{m}), 6.44(1 \mathrm{H}$, $\left.\mathrm{dd},{ }^{3} J=7.8 \mathrm{~Hz},{ }^{4} J=1.0 \mathrm{~Hz}\right), 6.56\left(1 \mathrm{H}, \mathrm{d},{ }^{3} J=7.3 \mathrm{~Hz}\right), 6.67(1 \mathrm{H}, \mathrm{s})$, $7.04\left(1 \mathrm{H}, \mathrm{t},{ }^{3} \mathrm{~J}=7.7 \mathrm{~Hz}\right)$. (Three $\mathrm{NH}$ protons were not assigned). ${ }^{13} \mathrm{C}$ NMR $\left(\mathrm{CDCl}_{3}, 298 \mathrm{~K}\right) \delta_{\mathrm{c}} \mathrm{ppm}: 32.1$ (1C), $39.8(1 \mathrm{C}), 41.7$ (1C), 54.2 (2C), 60.7 (1C), 69.5 (2C), 69.9 (2C), 70.0 (2C), $70.6(2 \mathrm{C}), 110.7$ (1C), 113.7 (1C), 117.7 (1C), 128.8 (1C), 140.0 (1C), 148.5 (1C).

$N^{I}-(3-((1,4,7,10,13-P e n t a o x a-16$-azacyclooctadecan-16-yl)methyl)phenyl)propane-1,3-diamine (7a). Obtained from compound 2 (0.9 mmol, $389 \mathrm{mg}$ ), propane-1,3-diamine (5a) (3.6 mmol, $266 \mathrm{mg})$, in the presence of $\mathrm{Pd}(\mathrm{dba})_{2}(4 \mathrm{~mol} \%, 21 \mathrm{mg})$, BINAP $(4.5 \mathrm{~mol} \%, 25 \mathrm{mg}), t \mathrm{BuONa}(1.4 \mathrm{mmol}, 134 \mathrm{mg})$ in $9 \mathrm{ml}$ dioxane. Eluent: $\mathrm{CH}_{2} \mathrm{Cl}_{2} / \mathrm{MeOH}$ (3:1). Yield $178 \mathrm{mg}$ (46\%). (MALDI-TOF) found: 426.2995. $\mathrm{C}_{22} \mathrm{H}_{40} \mathrm{~N}_{3} \mathrm{O}_{5}$ requires $426.2968[\mathrm{M}+\mathrm{H}]^{+} .{ }^{1} \mathrm{H}$ NMR $\left(\mathrm{CDCl}_{3}, 298 \mathrm{~K}\right) \delta_{\mathrm{H}}$ ppm: $1.79\left(2 \mathrm{H}\right.$, quintet, $\left.{ }^{3} J=6.5 \mathrm{~Hz}\right), 2.72(4 \mathrm{H}, \mathrm{t}$, $\left.{ }^{3} J=5.6 \mathrm{~Hz}\right), 2.87\left(2 \mathrm{H}, \mathrm{t},{ }^{3} J=6.6 \mathrm{~Hz}\right), 3.14\left(2 \mathrm{H}, \mathrm{t},{ }^{3} J=6.4 \mathrm{~Hz}\right), 3.52$ $(2 \mathrm{H}, \mathrm{s}), 3.55-3.65(20 \mathrm{H}, \mathrm{m}), 6.43\left(1 \mathrm{H}, \mathrm{dd},{ }^{3} J=7.8 \mathrm{~Hz},{ }^{4} J=1.1 \mathrm{~Hz}\right)$, $6.53\left(1 \mathrm{H}, \mathrm{d},{ }^{3} J=7.5 \mathrm{~Hz}\right), 6.59(1 \mathrm{H}, \mathrm{s}), 7.01\left(1 \mathrm{H}, \mathrm{t},{ }^{3} J=7.8 \mathrm{~Hz}\right)$. (Three NH protons were not assigned). ${ }^{13} \mathrm{C}$ NMR $\left(\mathrm{CDCl}_{3}, 298 \mathrm{~K}\right)$ $\delta_{\mathrm{c}} \mathrm{ppm}: 31.0(1 \mathrm{C}), 39.3$ (1C), 41.4 (1C), 54.0 (2C), 59.6 (1C), 69.6 (2C), 70.2 (2C), 70.5 (4C), 70.7 (2C), 110.9 (1C), 113.5 (1C), 117.5 (1C), 128.8 (1C), 140.0 (1C), 148.5 (1C).

$N^{1}, N^{3}$-bis $(3-((1,4,7,10,13-P e n t a o x a-16-a z a c y c l o o c t a d e c a n-$ 16-yl)methyl)phenyl)propane-1,3-diamine ( $8 \boldsymbol{a}$ ) was obtained as the second product in the synthesis of compound $7 \mathbf{a}$ from compound 2 (0.92 mmol, $396 \mathrm{mg}$ ), propane-1,3-diamine (5a) (2.7 mmol, $200 \mathrm{mg})$, in the presence of $\operatorname{Pd}(\mathrm{dba}),(4 \mathrm{~mol} \%, 21 \mathrm{mg})$, BINAP $(4.5 \mathrm{~mol} \%, 26 \mathrm{mg}), t \mathrm{BuONa}(1.4 \mathrm{mmol}, 134 \mathrm{mg})$ in $10 \mathrm{ml}$ dioxane. $\mathrm{CH}_{2} \mathrm{Cl}_{2} / \mathrm{MeOH} / \mathrm{NH}_{3}$ aq (100:35:6). Yield $30 \mathrm{mg}$ (8\%). (MALDITOF) found: 777.48. $\mathrm{C}_{41} \mathrm{H}_{69} \mathrm{~N}_{4} \mathrm{O}_{10}$ requires $777.50[\mathrm{M}+\mathrm{H}]^{+}$. ${ }^{1} \mathrm{H} \mathrm{NMR}$ $\left(\mathrm{CDCl}_{3}, 298 \mathrm{~K}\right) \delta_{\mathrm{H}} \mathrm{ppm}: 1.90\left(2 \mathrm{H}\right.$, quintet, $\left.{ }^{3} \mathrm{~J}=6.7 \mathrm{~Hz}\right), 2.75(8 \mathrm{H}$, t, $\left.{ }^{3} \mathrm{~J}=5.6 \mathrm{~Hz}\right), 3.22\left(4 \mathrm{H}, \mathrm{t},{ }^{3} \mathrm{~J}=6.6 \mathrm{~Hz}\right), 3.55-3.67(44 \mathrm{H}, \mathrm{m}), 3.90$ $\left(2 \mathrm{H}\right.$, br. s), $6.46\left(2 \mathrm{H}, \mathrm{d},{ }^{3} J=7.7 \mathrm{~Hz}\right), 6.60\left(2 \mathrm{H}, \mathrm{d},{ }^{3} J=7.5 \mathrm{~Hz}\right), 6.65$ $(2 \mathrm{H}, \mathrm{s}), 7.06\left(2 \mathrm{H}, \mathrm{t},{ }^{3} J=7.5 \mathrm{~Hz}\right) .{ }^{13} \mathrm{C} \mathrm{NMR}\left(\mathrm{CDCl}_{3}, 298 \mathrm{~K}\right) \delta_{\mathrm{c}} \mathrm{ppm}$ :
29.2 (1C), 41.9 (2C), 54.0 (4C), 60.0 (2C), 69.8 (4C), 70.2 (4C), 70.6 (8C), 70.7 (4C), 110.9 (2C), 113.5 (2C), 117.8 (2C), 128.9 (2C), $140.6(2 \mathrm{C}), 148.3(2 \mathrm{C})$.

3-((1,4, 7,10-Tetraoxa-13-azacyclopentadecan-13-yl)methyl)$\mathrm{N}$-(2-(2-(2-aminoethoxy)ethoxy)ethyl)-aniline (6b). Obtained from compound 3 (0.15 mmol, $66 \mathrm{mg})$, dioxadiamine (5b) $(0.3 \mathrm{mmol}$, $44 \mathrm{mg})$, in the presence of CuI $(10 \mathrm{~mol} \%, 3 \mathrm{mg}), l$-proline $(20$ $\mathrm{mol} \%, 3.5 \mathrm{mg}), \mathrm{Cs}_{2} \mathrm{CO}_{3}(0.225 \mathrm{mmol}, 73 \mathrm{mg})$ in $1 \mathrm{ml} \mathrm{EtCN}$. Eluent: $\mathrm{CH}_{2} \mathrm{Cl}_{2} / \mathrm{MeOH}$ (3:1), $\mathrm{CH}_{2} \mathrm{Cl}_{2} / \mathrm{MeOH} / \mathrm{NH}_{3}$ aq (100:20:1-100:20:3). Yield $51 \mathrm{mg}$ (75\%). (MALDI-TOF) found: 456.3132. $\mathrm{C}_{23} \mathrm{H}_{42} \mathrm{~N}_{3} \mathrm{O}_{6}$ requires $456.3074[\mathrm{M}+\mathrm{H}]^{+} .{ }^{1} \mathrm{H} \mathrm{NMR}\left(\mathrm{CDCl}_{3}, 298 \mathrm{~K}\right) \delta_{\mathrm{H}} \mathrm{ppm:} 3.03$ (2H, br. s), $3.19(4 \mathrm{H}, \mathrm{bs}), 3.35\left(2 \mathrm{H}, \mathrm{t},{ }^{3} J=4.8 \mathrm{~Hz}\right), 3.59-3.70(20 \mathrm{H}$, $\mathrm{m}), 3.74\left(2 \mathrm{H}, \mathrm{t},{ }^{3} \mathrm{~J}=4.8 \mathrm{~Hz}\right), 3.82\left(4 \mathrm{H}, \mathrm{t},{ }^{3} \mathrm{~J}=4.7 \mathrm{~Hz}\right), 4.02(1 \mathrm{H}$, bs), 6.56-6.63 $(2 \mathrm{H}, \mathrm{m}), 7.14\left(1 \mathrm{H}, \mathrm{t},{ }^{3} J=7.7 \mathrm{~Hz}\right), 7.22(1 \mathrm{H}, \mathrm{bs}) .\left(\mathrm{NH}_{2}\right.$ protons were not assigned). ${ }^{13} \mathrm{C} \mathrm{NMR}\left(\mathrm{CDCl}_{3}, 298 \mathrm{~K}\right) \delta_{\mathrm{c}} \mathrm{ppm}: 39.5$ (1C), 43.3 (1C), 53.7 (2C), 56.7 (1C), 66.5 (2C), 68.4-70.1 (10C, $\mathrm{m}), 112.0$ (1C), 116.2 (1C), 119.3 (1C), 129.5 (1C), 136.9 (1C), $149.0(1 \mathrm{C})$.

3-((1,4,7,10-Tetraoxa-13-azacyclopentadecan-13-yl)methyl)$\mathrm{N}$-(2-(2-(2-(2-aminoethoxy)ethoxy)-ethoxy)ethyl)aniline (6c). Obtained from compound $3(0.15 \mathrm{mmol}, 66 \mathrm{mg})$, trioxadiamine (5c) $(0.3 \mathrm{mmol}, 58 \mathrm{mg})$, in the presence of $\mathrm{CuI}(10 \mathrm{~mol} \%, 3 \mathrm{mg})$, l-proline $(20 \mathrm{~mol} \%, 3.5 \mathrm{mg}), \mathrm{Cs}_{2} \mathrm{CO}_{3}(0.225 \mathrm{mmol}, 73 \mathrm{mg})$ in $1 \mathrm{ml}$ EtCN. Eluent: $\mathrm{CH}_{2} \mathrm{Cl}_{2} / \mathrm{MeOH}(3: 1)$. Yield $25 \mathrm{mg}$ (33\%). (MALDITOF) found: $500.3301 . \mathrm{C}_{25} \mathrm{H}_{46} \mathrm{~N}_{3} \mathrm{O}_{7}$ requires $500.3336[\mathrm{M}+\mathrm{H}]^{+}$. ${ }^{1} \mathrm{H}$ NMR $\left(\mathrm{CDCl}_{3}, 298 \mathrm{~K}\right) \delta_{\mathrm{H}}$ ppm: $2.81(2 \mathrm{H}, \mathrm{bs}), 2.87$ (4H, br. s), $3.25\left(2 \mathrm{H}, \mathrm{t},{ }^{3} \mathrm{~J}=4.7 \mathrm{~Hz}\right), 3.55-3.69(26 \mathrm{H}, \mathrm{m}), 3.71\left(2 \mathrm{H}, \mathrm{t},{ }^{3} J=5.1\right.$ $\mathrm{Hz}), 3.78\left(2 \mathrm{H}, \mathrm{t},{ }^{3} J=5.1 \mathrm{~Hz}\right), 4.12(1 \mathrm{H}, \mathrm{bs}), 6.53\left(2 \mathrm{H}, \mathrm{d},{ }^{3} J=8.0\right.$ $\mathrm{Hz}), 7.09\left(1 \mathrm{H}, \mathrm{t},{ }^{3} \mathrm{~J}=7.7 \mathrm{~Hz}\right), 7.09(1 \mathrm{H}, \mathrm{s}) .\left(\mathrm{NH}_{2}\right.$ protons were not assigned). ${ }^{13} \mathrm{C} \mathrm{NMR}\left(\mathrm{CDCl}_{3}, 298 \mathrm{~K}\right) \delta_{\mathrm{c}} \mathrm{ppm}: 39.6(1 \mathrm{C}), 43.6$ (1C), 53.9 (2C), 58.7 (1C), 66.3 (2C), 68.2 (1C), 68.9-70.4 (11C, $\mathrm{m}), 111.6$ (1C), 116.3 (1C), 119.5 (1C), 129.3 (1C), 136.6 (1C), $149.0(1 \mathrm{C})$.

3-((1,4,7,10-Tetraoxa-13-azacyclopentadecan-13-yl)methyl)$\mathrm{N}$-(3-(2-(2-(3-aminopropoxy)ethoxy)-ethoxy)propyl)aniline (6d). Obtained from compound $3(0.29 \mathrm{mmol}, 128 \mathrm{mg})$, trioxadiamine (5d) $(0.58 \mathrm{mmol}, 128 \mathrm{mg})$, in the presence of CuI $(10 \mathrm{~mol} \%, 5.5 \mathrm{mg})$, l-proline $(20 \mathrm{~mol} \%, 6.7 \mathrm{mg}), \mathrm{Cs}_{2} \mathrm{CO}_{3}(0.435 \mathrm{mmol}, 142 \mathrm{mg})$ in 2 ml EtCN. Eluent: $\mathrm{CH}_{2} \mathrm{Cl}_{2} / \mathrm{MeOH}$ (5:1-3:1). Yield $57 \mathrm{mg}$ (37\%). (MALDI-TOF) found: $528.3577 . \mathrm{C}_{27} \mathrm{H}_{50} \mathrm{~N}_{3} \mathrm{O}_{7}$ requires 528.3649 $[\mathrm{M}+\mathrm{H}]^{+} .{ }^{1} \mathrm{H} \mathrm{NMR}\left(\mathrm{CD}_{3} \mathrm{OD}, 298 \mathrm{~K}\right) \delta_{\mathrm{H}} \mathrm{ppm}: 1.88\left(4 \mathrm{H}\right.$, quintet, ${ }^{3} \mathrm{~J}$ $=6.1 \mathrm{~Hz}), 2.72\left(4 \mathrm{H}, \mathrm{t},{ }^{3} \mathrm{~J}=4.5 \mathrm{~Hz}\right), 3.03(2 \mathrm{H}, \mathrm{bs}), 3.19\left(2 \mathrm{H}, \mathrm{t},{ }^{3} J=\right.$ $6.6 \mathrm{~Hz}), 3.56-3.72(30 \mathrm{H}, \mathrm{m}), 6.55\left(1 \mathrm{H}, \mathrm{d},{ }^{3} J=7.1 \mathrm{~Hz}\right), 6.59(1 \mathrm{H}$, s), $6.60\left(1 \mathrm{H}, \mathrm{d},{ }^{3} J=8.0 \mathrm{~Hz}\right), 7.11\left(1 \mathrm{H}, \mathrm{t},{ }^{3} J=7.5 \mathrm{~Hz}\right)$. (NH protons were not observed). ${ }^{13} \mathrm{C}$ NMR $\left(\mathrm{CD}_{3} \mathrm{OD}, 298 \mathrm{~K}\right) \delta_{\mathrm{c}} \mathrm{ppm}: 28.9(1 \mathrm{C})$, 29.4 (1C), 38.6 (1C), 40.8 (1C), 53.0 (2C), 57.7 (1C), 66.9 (2C), 68.0 (1C), 68.5-70.1 (9C, m), 111.8 (1C), 114.8 (1C), $118.6(1 \mathrm{C})$, 129.0 (1C), 136. 2 (1C), 149.1 (1C).

$3-((1,4,7,10,13-P e n t a o x a-16-a z a c y c l o o c t a d e c a n-16-y l)$ methyl)-N-(3-(2-(2-(3-aminopropoxy)ethoxy)-ethoxy)propyl) aniline $(7 \boldsymbol{d})$. Obtained from compound $4(0.5 \mathrm{mmol}, 222 \mathrm{mg})$, trioxadiamine $(\mathbf{5 d})(1 \mathrm{mmol}, 220 \mathrm{mg})$, in the presence of $\mathrm{CuI}$ $(10 \mathrm{~mol} \%$, $9.5 \mathrm{mg})$, l-proline (20 mol\%, $10.5 \mathrm{mg}), \mathrm{Cs}_{2} \mathrm{CO}_{3}$ (1.5 mmol, $489 \mathrm{mg})$ in $2 \mathrm{ml} \mathrm{EtCN}$. Eluent: $\mathrm{CH}_{2} \mathrm{Cl}_{2} / \mathrm{MeOH} / \mathrm{NH}_{3} \mathrm{aq}$ (100:20:1-100:20:3). Yield $185 \mathrm{mg}$ (65 \%). (MALDI-TOF) found: 572.3950. $\mathrm{C}_{29} \mathrm{H}_{54} \mathrm{~N}_{3} \mathrm{O}_{8}$ requires $572.3911[\mathrm{M}+\mathrm{H}]^{+} .{ }^{1} \mathrm{H} \mathrm{NMR}\left(\mathrm{CDCl}_{3}\right.$, $328 \mathrm{~K}) \delta_{\mathrm{H}}$ ppm: $1.76\left(2 \mathrm{H}\right.$, quintet, $\left.{ }^{3} J=6.8 \mathrm{~Hz}\right), 1.83(2 \mathrm{H}$, quintet, $\left.{ }^{3} J=6.0 \mathrm{~Hz}\right), 2.85-2.94(6 \mathrm{H}, \mathrm{m}), 3.18\left(2 \mathrm{H}, \mathrm{t},{ }^{3} J=6.4 \mathrm{~Hz}\right), 3.50$ $\left(2 \mathrm{H}, \mathrm{t},{ }^{3} J=5.7 \mathrm{~Hz}\right), 3.50-3.64(28 \mathrm{H}, \mathrm{m}), 3.69(4 \mathrm{H}, \mathrm{bs}), 6.47(1 \mathrm{H}$, $\left.\mathrm{d},{ }^{3} J=7.5 \mathrm{~Hz}\right), 6.50\left(1 \mathrm{H}, \mathrm{d},{ }^{3} J=8.0 \mathrm{~Hz}\right), 6.65(1 \mathrm{H}, \mathrm{s}), 7.02(1 \mathrm{H}, \mathrm{t}$, $\left.{ }^{3} J=7.7 \mathrm{~Hz}\right)$. (NH protons were not assigned). ${ }^{13} \mathrm{C} \mathrm{NMR}\left(\mathrm{CDCl}_{3}\right.$, $298 \mathrm{~K}) \delta_{c}$ ppm: 27.9 (1C), 29,2 (1C), 37.7 (1C), 41.6 (1C), 54.2 (2C), 57.0 (1C), 66.9 (2C), 68.1 (1C), 69.5-70.5 (13C, m), 112.0 (1C), 114.8 (1C), 118.1 (1C), 129.2 (1C), 149.2 (1C). (One aromatic quaternary carbon was not assigned).

$N^{1}, N^{I^{\prime}}-\left(3,3^{\prime}-(1,4,10,13-T e t r a o x a-7,16-d i a z a c y c l o o c t a d e c a n e-\right.$ 7,16-diyl)bis (methylene)bis(3,1-phenylene))dipropane-1,3diamine (10a). Obtained from compound $9(0.15 \mathrm{mmol}, 104 \mathrm{mg})$, 
propane-1,3-diamine $\mathbf{( 5 a})(0.6 \mathrm{mmol}, 44 \mathrm{mg})$, in the presence of $\mathrm{CuI}$ (20 mol\%, $6 \mathrm{mg}$ ), $l$-proline ( $40 \mathrm{~mol} \%, 7 \mathrm{mg}$ ), $\mathrm{Cs}_{2} \mathrm{CO}_{3}(0.45 \mathrm{mmol}$, $147 \mathrm{mg})$ in $1 \mathrm{ml} \mathrm{EtCN}$. Eluent: $\mathrm{CH}_{2} \mathrm{Cl}_{2} / \mathrm{MeOH} / \mathrm{NH}_{3}$ aq $(100: 35: 6-$ 10:4:1). Yield $52 \mathrm{mg}(36 \%)$. (MALDI-TOF) found: 587.4349 . $\mathrm{C}_{32} \mathrm{H}_{55} \mathrm{~N}_{6} \mathrm{O}_{4}$ requires $587.4285[\mathrm{M}+\mathrm{H}]^{+}$. ${ }^{1} \mathrm{H} \mathrm{NMR}\left(\mathrm{CD}_{3} \mathrm{OD}, 298 \mathrm{~K}\right)$ $\delta_{\mathrm{H}}$ ppm: $1.79\left(4 \mathrm{H}\right.$, quintet, $\left.{ }^{3} \mathrm{~J}=7.0 \mathrm{~Hz}\right), 2.71\left(8 \mathrm{H}, \mathrm{t},{ }^{3} \mathrm{~J}=4.9 \mathrm{~Hz}\right)$, $2.81\left(4 \mathrm{H}, \mathrm{t},{ }^{3} J=6.5 \mathrm{~Hz}\right), 3.17\left(4 \mathrm{H}, \mathrm{t},{ }^{3} J=6.5 \mathrm{~Hz}\right), 3.51(4 \mathrm{H}, \mathrm{s}), 3.54$ $(8 \mathrm{H}, \mathrm{s}), 3.61\left(8 \mathrm{H}, \mathrm{t},{ }^{3} J=4.9 \mathrm{~Hz}\right), 6.47\left(2 \mathrm{H}, \mathrm{d},{ }^{3} J=7.2 \mathrm{~Hz}\right), 6.53$ $(2 \mathrm{H}, \mathrm{s}), 6.54\left(2 \mathrm{H}, \mathrm{d},{ }^{3} J=7.6 \mathrm{~Hz}\right), 7.06\left(2 \mathrm{H}, \mathrm{t},{ }^{3} J=7.7 \mathrm{~Hz}\right)$. (NH protons were not assigned). ${ }^{13} \mathrm{C}$ NMR $\left(\mathrm{CD}_{3} \mathrm{OD}, 298 \mathrm{~K}\right) \delta_{c} \mathrm{ppm}$ : 31.8 (2C), 39.9 (2C), 42.0 (2C), 55.2 (4C), 58.6 (2C), 69.8 (4C), 71.3 (4C), 112.5 (2C), 115.7 (2C), 119.4 (2C), 130.0 (2C), 139.3 (2C), $150.0(2 \mathrm{C})$.

3,3'-(1,4,10,13-Tetraoxa-7,16-diazacyclooctadecane-7,16diyl)bis(methylene)bis(N-(2-(2-(2-(2-aminoethoxy)ethoxy)ethoxy) ethyl)aniline) (10d). Obtained from compound 9 (0.5 mmol, $347 \mathrm{mg}$ ), trioxadiamine (5d) $(2 \mathrm{mmol}, 440 \mathrm{mg})$, in the presence of $\mathrm{CuI}(20 \mathrm{~mol} \%, 20 \mathrm{mg}), l$-proline $(40 \mathrm{~mol} \%, 24 \mathrm{mg}), \mathrm{Cs}_{2} \mathrm{CO}_{3}$ $(2 \mathrm{mmol}, 652 \mathrm{mg})$ in $1 \mathrm{ml}$ EtCN. Yield in the reaction mixture 76\%. (MALDI-TOF) found: 823.58. $\mathrm{C}_{42} \mathrm{H}_{75} \mathrm{~N}_{6} \mathrm{O}_{10}$ requires 823.55 $[\mathrm{M}+\mathrm{H}]^{+} .{ }^{1} \mathrm{H}$ NMR $\left(\mathrm{CDCl}_{3}, 298 \mathrm{~K}\right) \delta_{\mathrm{H}} \mathrm{ppm}: 1.63(4 \mathrm{H}, \mathrm{bs}), 1.79$ $\left(4 \mathrm{H}\right.$, quintet, $\left.{ }^{3} \mathrm{~J}=6.1 \mathrm{~Hz}\right), 2.66(8 \mathrm{H}, \mathrm{bs}), 2.70(4 \mathrm{H}, \mathrm{bs}), 3.10(4 \mathrm{H}$, $\left.\mathrm{t},{ }^{3} \mathrm{~J}=6.1 \mathrm{~Hz}\right), 3.40-3.56(32 \mathrm{H}, \mathrm{m}), 6.38\left(2 \mathrm{H}, \mathrm{d},{ }^{3} J=7.7 \mathrm{~Hz}\right), 6.46$ $\left(2 \mathrm{H}, \mathrm{d},{ }^{3} J=6.8 \mathrm{~Hz}\right), 6.52(2 \mathrm{H}, \mathrm{s}), 6.98\left(2 \mathrm{H}, \mathrm{t},{ }^{3} J=7.7 \mathrm{~Hz}\right) .(\mathrm{NH}$ protons were not assigned).

3-((1,4,7,10-Tetraoxa-13-azacyclopentadecan-13-yl)methyl)N-(3-(2-(2-(3-(4-((1,4,7,10-tetraoxa-13-azacyclopentadecan-13yl)methyl)phenylamino) propoxy)ethoxy)ethoxy)propyl)aniline (11d). Obtained from compound $3(0.78 \mathrm{mmol}, 340 \mathrm{mg})$, trioxadiamine (5d) (0.39 mmol, $85 \mathrm{mg})$, in the presence of CuI (20 mol\%, $15 \mathrm{mg}$ ), 2-(isobutyryl)cyclohexanone (40 mol\%, $26 \mathrm{mg}), \mathrm{Cs}_{2} \mathrm{CO}_{3}$ ( $1.5 \mathrm{mmol}, 489 \mathrm{mg})$ in $1 \mathrm{ml}$ DMF. Eluent: $\mathrm{CH}_{2} \mathrm{Cl}_{2} / \mathrm{MeOH} / \mathrm{NH}_{3} \mathrm{aq}$ (100:20:2). Yield $92 \mathrm{mg}$ (28\%). (MALDI-TOF) found: 835.5380. $\mathrm{C}_{44} \mathrm{H}_{75} \mathrm{~N}_{4} \mathrm{O}_{11}$ requires $835.5432[\mathrm{M}+\mathrm{H}]^{+}$. ${ }^{1} \mathrm{H} \mathrm{NMR}\left(\mathrm{CDCl}_{3}, 298 \mathrm{~K}\right)$ $\delta_{\mathrm{H}}$ ppm: $1.86\left(4 \mathrm{H}\right.$, quintet, $\left.{ }^{3} J=6.3 \mathrm{~Hz}\right), 2.77\left(8 \mathrm{H}, \mathrm{t},{ }^{3} J=5.8 \mathrm{~Hz}\right)$, $3.19\left(4 \mathrm{H}, \mathrm{t},{ }^{3} J=6.6 \mathrm{~Hz}\right), 6.44\left(2 \mathrm{H}, \mathrm{dd},{ }^{3} J=7.8 \mathrm{~Hz},{ }^{4} J=1.3 \mathrm{~Hz}\right)$, $6.59\left(2 \mathrm{H}, \mathrm{d},{ }^{3} J=7.6 \mathrm{~Hz}\right), 6.62(2 \mathrm{H}, \mathrm{s}), 7.05\left(2 \mathrm{H}, \mathrm{t},{ }^{3} J=7.8 \mathrm{~Hz}\right)$. (NH protons were not assigned). ${ }^{13} \mathrm{C} \mathrm{NMR}\left(\mathrm{CDCl}_{3}, 298 \mathrm{~K}\right) \delta_{\mathrm{c}} \mathrm{ppm}$ : 29.2 (2C), 41.6 (2C), 54.3 (4C), 60.8 (2C), 69.6 (2C), 69.8 (4C), 70.1 (4C), 70.2 (2C), 70.4 (4C), 70.6 (2C), 70.8 (4C), 111.0 (2C), 113.4 (2C), $117.6(2 \mathrm{C}), 128.9(2 \mathrm{C}), 140.4$ (2C), 148.6 (2C).

$N, N^{\prime}-\left(3,3^{\prime}-\left(2,2^{\prime}\right.\right.$-Oxybis (ethane-2,1-diyl)bis(oxy)) bis(propane-3,1-diyl))bis(3-((16-(3-iodobenzyl)-1,4,10,13tetraoxa-7,16-diazacyclooctadecan-7-yl)methyl)aniline) (12d). Obtained from compound 9 (1 mmol, $694 \mathrm{mg})$, trioxadiamine (5d) $(0.5 \mathrm{mmol}, 110 \mathrm{mg})$, in the presence of $\mathrm{CuI}(20 \mathrm{~mol} \%$, $19 \mathrm{mg}$ ), 2-(isobutyryl)cyclohexanone (40 mol\%, $34 \mathrm{mg}$ ), $\mathrm{Cs}_{2} \mathrm{CO}_{3}$ $(1.5 \mathrm{mmol}, 489 \mathrm{mg})$ in $1 \mathrm{ml}$ DMF. Eluent: $\mathrm{CH}_{2} \mathrm{Cl}_{2} / \mathrm{MeOH} / \mathrm{NH}_{3} \mathrm{aq}$ (100:25:1-100:25:2). Yield $120 \mathrm{mg}$ (18 \%). (MALDI-TOF) found: 1353.54. $\mathrm{C}_{62} \mathrm{H}_{95} \mathrm{I}_{2} \mathrm{~N}_{6} \mathrm{O}_{11}$ requires $1353.51[\mathrm{M}+\mathrm{H}]^{+} .{ }^{1} \mathrm{H} \mathrm{NMR}$ $\left(\mathrm{CDCl}_{3}, 298 \mathrm{~K}\right) \delta_{\mathrm{H}}$ ppm: $1.86\left(4 \mathrm{H}\right.$, quintet, $\left.{ }^{3} J=5.9 \mathrm{~Hz}\right), 2.74-2.86$ $(16 \mathrm{H}, \mathrm{m}), 3.19\left(4 \mathrm{H}, \mathrm{t},{ }^{3} \mathrm{~J}=6.4 \mathrm{~Hz}\right), 3.51-3.70(40 \mathrm{H}, \mathrm{m}), 6.45(2 \mathrm{H}$, d, $\left.{ }^{3} J=7.2 \mathrm{~Hz}\right), 6.58(2 \mathrm{H}, \mathrm{s}), 6.62\left(2 \mathrm{H}, \mathrm{d},{ }^{3} J=7.3 \mathrm{~Hz}\right), 7.00(2 \mathrm{H}$, t, $\left.{ }^{3} J=7.6 \mathrm{~Hz}\right), 7.06\left(2 \mathrm{H}, \mathrm{t},{ }^{3} J=7.5 \mathrm{~Hz}\right), 7.29\left(2 \mathrm{H}, \mathrm{d},{ }^{3} J=7.3 \mathrm{~Hz}\right)$, $7.53\left(2 \mathrm{H}, \mathrm{d},{ }^{3} \mathrm{~J}=7.3 \mathrm{~Hz}\right), 7.69(2 \mathrm{H}, \mathrm{s})$. (NH protons were not assigned). ${ }^{13} \mathrm{C} \mathrm{NMR}\left(\mathrm{CDCl}_{3}, 298 \mathrm{~K}\right) \delta_{\mathrm{c}} \mathrm{ppm}: 29.2(2 \mathrm{C}), 41.6(2 \mathrm{C})$, 53.6 (4C), 53.7 (4C), 59.2 (2C), 60.0 (2C), 69.7 (2C), 69.9 (8C), 70.2 (2C), 70.6 (8C), 94.3 (1C), 111.2 (2C), 113.3 (2C), 117.7 (2C), 127.9 (2C), 128.9 (2C), 129.9 (2C), 135.8 (2C), 137.5 (2C), 141.8 (2C), 142.4 (2C), 148.5 (2C).

\section{Results and Discussion}

Initially 1-aza-15-crown-5 and 1-aza-18-crown-6 were modified with 3-bromobenzyl or 3-iodobenzyl substituents using simple nucleophilic substitution reactions with corresponding benzyl bromides (Scheme 1). Target products 1-4 were obtained in high yields (92-96\%) after simple work-up of the reaction mixtures.

First bromobenzyl derivatives of azacrown ethers $\mathbf{1}$ and $\mathbf{2}$ were employed in the $\operatorname{Pd}(0)$-catalyzed reactions with 3 equiv. of propane-1,3-diamine 5a to check the selectivity of the $N$-monoarylation $v s N, N$ '-diarylation and to elaborate the conditions for the chromatographic isolation of the target products. Standard conditions for these processes were applied: $\mathrm{Pd}(\mathrm{dba})_{2} / \mathrm{BINAP}$ catalytic system (4/4.5 mol\%), 1.5 equiv. $t \mathrm{BuONa}$ as a base. The amination of compound 1 using $0.02 \mathrm{M}$ solution of $\mathbf{1}$ led to $85 \%$ yield of the monoarylation product 6a, and after column chromatography it was isolated in $43 \%$ yield, whereas the same reaction in a more concentrated solution $(C=0.05 \mathrm{M})$ led to $87 \%$ yield of the target compound $\mathbf{6 a}$ in the reaction mixture and $58 \%$ yield after chromatography. In the case of compound $\mathbf{2}$ the similar reaction led to $77 \%$ yield of the product of monoarylation $7 \mathbf{a}$ and $23 \%$ yield of the diarylated diamine 8a. After column chromatography their yields were 30 and $8 \%$ respectively (Scheme 2). The application of 4 equiv. of propane-1,3diamine in the reaction with compound 2 resulted in $88 \%$ yield of the target compound $7 \mathbf{a}$ in the reaction mixture, thus it can be used in situ for some further transformations. The yield after chromatography in this case also increased to $46 \%$. Amination reactions were also conducted with iodobenzyl derivative 4 using either $\mathrm{CuI} / \mathrm{Ll}$ (10/20 mol\%) catalytic system $(\mathrm{L} 1=l$-proline $), 1.5$ equiv. $\mathrm{Cs}_{2} \mathrm{CO}_{3}$ in boiling $\operatorname{EtCN}(C=0.5 \mathrm{M})$, or $\mathrm{CuI} / \mathrm{L} 2(10 / 20 \mathrm{~mol} \%)$ catalytic system ( $22=2$-isobutyrylcyclohexanone), 1.5 equiv. $\mathrm{Cs}_{2} \mathrm{CO}_{3}$ in DMF $\left(140{ }^{\circ} \mathrm{C}\right)$. The first reaction afforded $80 \%$ yield of the compound $\mathbf{7 a}$ in the reaction mixture and $22 \%$ after chromatographic isolation, the second reaction produced $17 \%$ of the same compound after chromatography. No product of diarylation 8a was noted in both cases. For this reason we chose $\mathrm{CuI} / \mathrm{L1} / \mathrm{EtCN}$ for further syntheses of monoaryl derivatives of polyoxadiamines. It should be noted that as the excess of propane-1,3-diamine can easily be removed in high vacuum and only 2 equiv. of this diamine

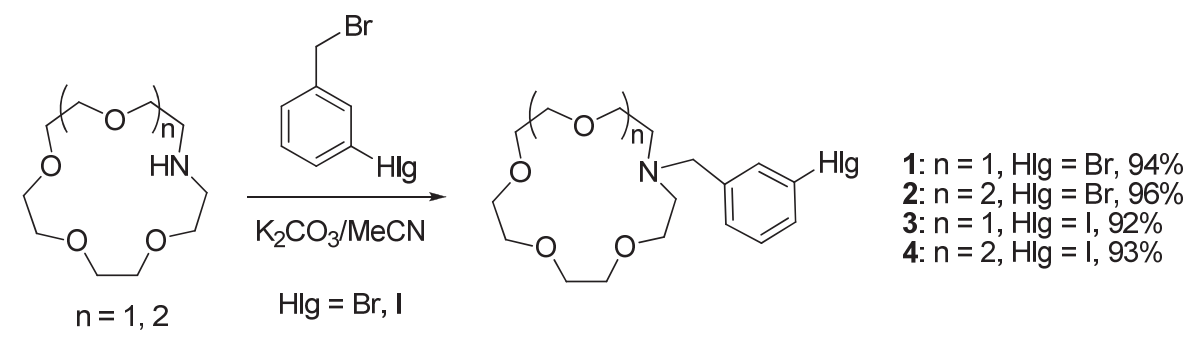

Scheme 1. 
<smiles>[Hg]c1cccc(CN2CCOCCOCCOCCOCC2)c1</smiles>

$1,2,4$

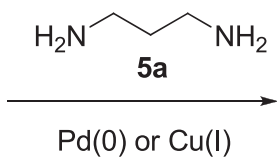

$\mathrm{Pd}(0)$ or $\mathrm{Cu}(\mathrm{I})$<smiles>NCCCNc1cccc(CN2CCOCCOCCOCCOCC2)c1</smiles>

6a: $n=1$

$7 a: n=2$<smiles>c1ccc(CN2CCOCCOCCOCCOCCOCC2)cc1</smiles>

Scheme 2.

are needed to form the monoarylated derivative $\mathbf{7 a}$, in the case of further transformations of this compound one does not need its chromatographic purification and may use it in situ.

Further $\mathrm{Cu}(\mathrm{I})$-catalyzed reactions were conducted using iodobenzyl derivatives $\mathbf{3}$ and $\mathbf{4}$ and a number of di- and trioxadiamines $\mathbf{5 b - c}$ taken in two-fold excess (Scheme 3). These reactions were catalyzed by $\mathrm{CuI} / \mathrm{L} 1(10 / 20 \mathrm{~mol} \%)$ in the presence of $\mathrm{Cs}_{2} \mathrm{CO}_{3}$ in EtCN and the products were isolated by column chromatography. Dioxadiamine $\mathbf{5 b}$ afforded $75 \%$ yield of the target compound $\mathbf{6 b}$, while the reactions with trioxadiamines $\mathbf{5 c , d}$ gave lower yields of corresponding derivatives $\mathbf{6 c , d}$ (33 and $37 \%$ ). The amination of compound 4 with trioxadiamine $\mathbf{5 d}$ was much more successful and the product 7d was obtained in $65 \%$ yield. No products of $N, N$ 'diarylation of polyoxadiamiens were isolated in all cases. This fact is in a good correspondence with our previous results of the $\mathrm{Cu}$-catalyzed arylation of polyoxadiamines. ${ }^{[27]}$ In this research we did not use polyamines as substrates though they were found to participate better than oxadiamines in the copper-catalyzed arylation and heteroarylation reactions. ${ }^{[27,28]}$ This was done in view of a practical impos- sibility of the isolation of their pure monoaryl derivatives bearing azacrown moiety from the reaction mixture with the excess of polyamine.

Next we investigated the possibility to introduce diazacrown ethers in the reactions with propane-1,3diamine and oxadiamines. $N, N$ '-di(3-iodobenzyl) derivative of diaza-18-crown-69 was synthesized in $90 \%$ yield, and it was introduced in the reaction with 4 equiv. of propane-1,3diamine catalyzed by CuI/L1 (20/40 mol\%) (Scheme 4). The yield of the target compound 10a exceeded $80 \%$ in the reaction mixture, and after column chromatography it was $36 \%$. The same reaction with trioxadiamine $\mathbf{5 d}$ afforded $76 \%$ yield of the bis(trioxadiamine) derivative $10 d$ in the reaction mixture.

According to our previous investigations, the coppercatalyzed $N, N$ '-diarylation of diamines and oxadiamines turned to be a challenging task. We carried out the diarylation of trioxadiamine 5d using 2 equiv. of iodobenzyl substituted azacrown 3 (Scheme 5). In this case the catalytic system $\mathrm{CuI} /$ L2 in DMF $\left(140^{\circ} \mathrm{C}\right)$ was employed because we had shown it to promote $N, N$ '-diarylation of oxadiamines with simple aryl

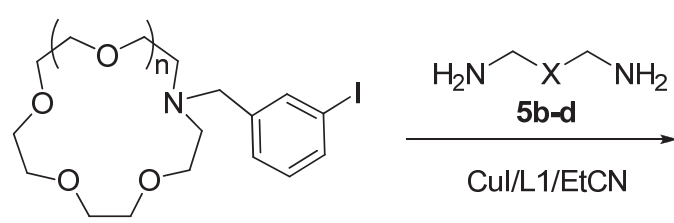

3,4

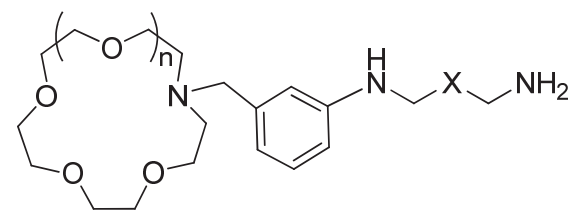

6b: $\mathrm{n}=1, \mathrm{X}=\mathrm{OCH}_{2} \mathrm{CH}_{2} \mathrm{O}, 75 \%$

6c: $n=1, X=\mathrm{OCH}_{2} \mathrm{CH}_{2} \mathrm{OCH}_{2} \mathrm{CH}_{2} \mathrm{O}, 33 \%$

6d: $n=1, X=\mathrm{CH}_{2} \mathrm{OCH}_{2} \mathrm{CH}_{2} \mathrm{OCH}_{2} \mathrm{CH}_{2} \mathrm{OCH}_{2}, 37 \%$

$7 d: n=2, X=\mathrm{CH}_{2} \mathrm{OCH}_{2} \mathrm{CH}_{2} \mathrm{OCH}_{2} \mathrm{CH}_{2} \mathrm{OCH}_{2}, 65 \%$

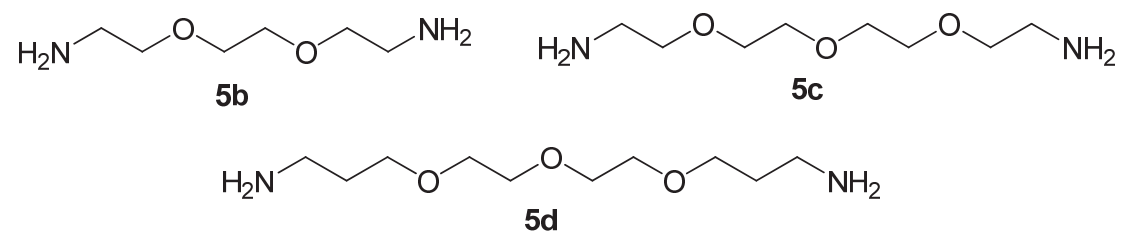

Scheme 3 . 

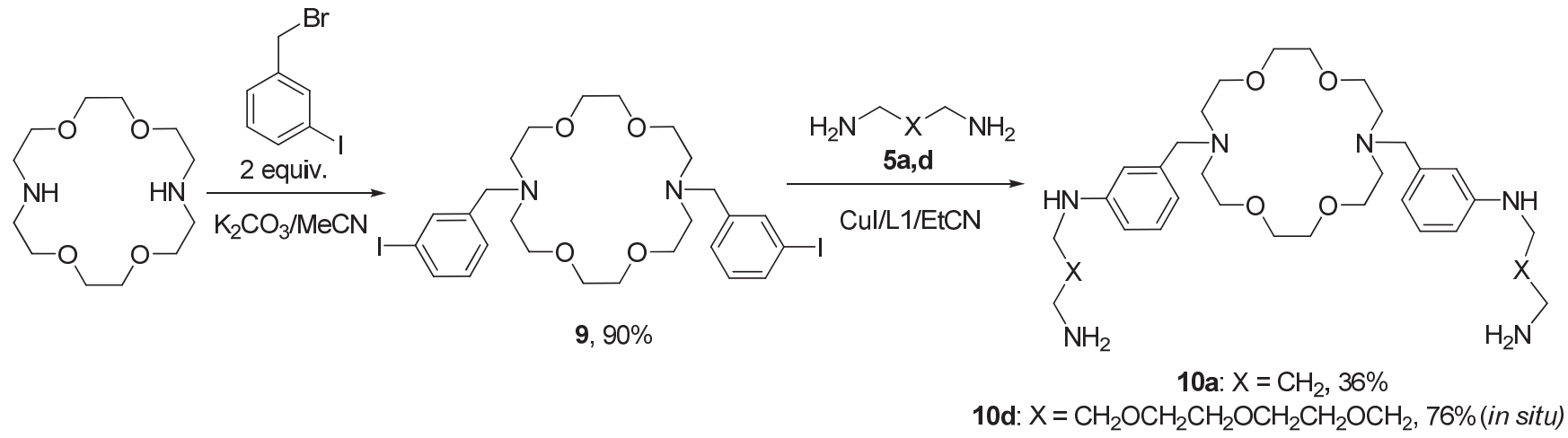

Scheme 4.
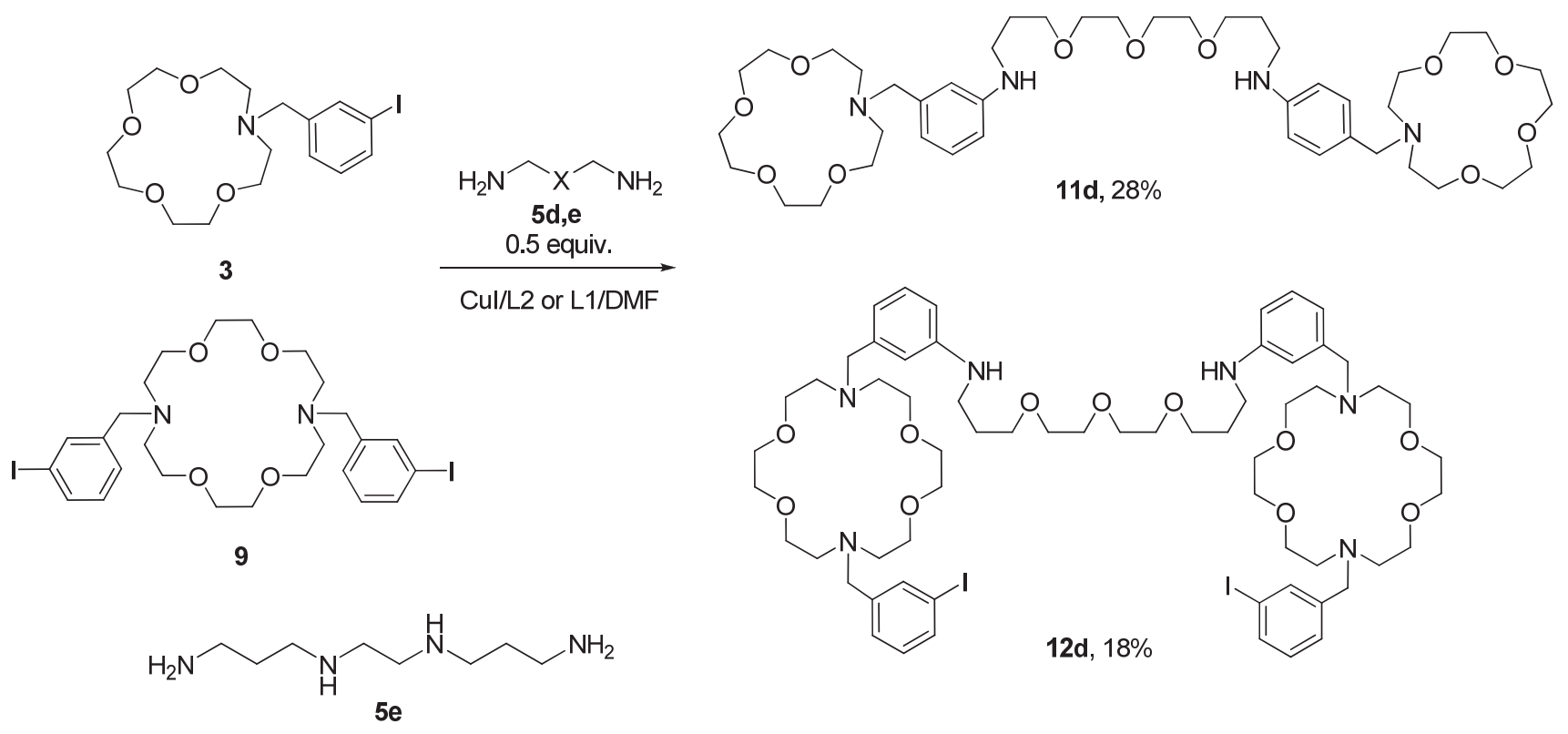

$11 d, 28 \%$

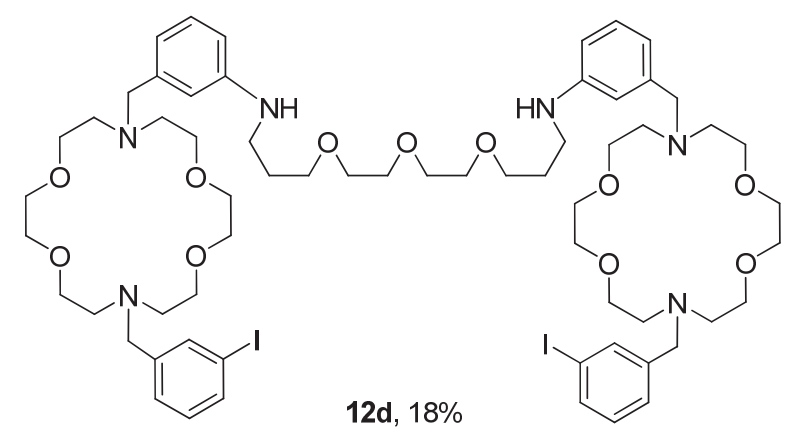

Scheme 5.

iodides. ${ }^{[27]}$ The target product $\mathbf{1 1 d}$ was isolated in $28 \%$ yield. The same reaction with tetraamine $\mathbf{5 e}$ catalyzed by $\mathrm{CuI} / \mathrm{L} 1$ in DMF gave a complex mixture of the products of arylation among which the target compound was detected by NMR and MALDI-TOF spectroscopy, however, the pure product could not be isolated by column chromatography, probably due to the formation of isomers with close $R_{\mathrm{f}}$. Similarly, the reaction of $N, N^{\prime}$-di(3-iodobenzyl) substituted diazacrown 9 produced corresponding bisazacrown derivative of trioxadiamine $\mathbf{1 2 d}$, but the reaction with tetraamine $5 \mathbf{e}$ gave only an inseparable mixture of unidentified compounds.

\section{Conclusions}

To sum up, we investigated the possibility to modify azacrown ethers with propane-1,3-diamine and polyoxadiamine podands using $\mathrm{Cu}(\mathrm{I})$-catalyzed amination and found out that this approach is quite applicable. The reactions mediated by $\mathrm{Cu}(\mathrm{I})$ and $\mathrm{Pd}(0)$ were shown to produce the target arylation products in comparable yields. Moderate yields of the target compounds were due to their difficult isolation using column chromatography, and in the case of propane-1,3-diamine its excess can be removed in vacuo, and the product was shown to be enough pure for further transformations. $\mathrm{Cu}(\mathrm{I})$-catalyzed diamination of the diazacrown derivative was also demonstrated as well as $N, N^{\prime}$-diarylation of trioxadiamine with iodobenzyl derivatives of aza- and diazacrown ethers. Only diamines and polyoxadiamines but not polyamines can be employed in these reactions.

Acknowledgements. This work was financially supported by the RFBR grants 12-03-00796, 12-03-93113, and by the Russian Academy of Sciences program P-8 "Development of the methods for the synthesis of new chemicals and creation of new materials".

\section{References}

1. Caceris W.P., Nickle S.K., Sherry A.D. Inorg. Chem. 1987, 26, 958-960.

2. Hama H., Takamoto S. Nippon Kagaku Kaishi 1975, 1182-1185. 
3. Stetter H., Frank W. Angew. Chem., Int. Ed. Engl. 1976, 15, 686-686.

4. Brucher E., Laurenczy G., Makra Z.S. Inorg. Chim. Acta 1987, 139, 141-142.

5. Medved T.Ya., Kabachnik M.I., Belskii F.I., Pisareva S.A. Izv. Akad. Nauk SSSR, Ser. Khim. 1988, 2103-2107 (in Russ.).

6. Polikarpov Yu.M., Belskii F.I., Pisareva S.A., Kabachnik M.I. Izv. Akad. Nauk SSSR, Ser. Khim. 1989, 2112-2116 (in Russ.).

7. Kabachnik M.I., Medved T.Ya., Belskii F.I., Pisareva S.A. Izv. Akad. Nauk SSSR, Ser. Khim. 1984, 844-849 (in Russ.).

8. Delgado R., Siegfried L.C., Kaden T.A. Helv. Chim. Acta 1990, 73, 140-148.

9. Kabachnik M.I., Polikarpov Yu.M. J. Gen. Chem. USSR (Engl. Transl.) 1988, 58, 1729-1750.

10. Pisareva S.A., Belskii F.I., Medved T.Ya., Kabachnik M.I. Izv. Akad. Nauk SSSR, Ser. Khim. 1987, 413-417 (in Russ.).

11. Kataky R., Matthes K.E., Nicholson P.E., Parker D., Buschmann H.J. J. Chem. Soc, Perkin Trans. 2 1990, 1425-1432.

12. Izatt R.M., Pawlak K., Bradshaw J.S. Chem. Rev. 1991, 91, 1721-2085.

13. Basak A.K., Kaden T.A. Helv. Chim. Acta 1983, 66, 20862092.

14. Evers A., Hancock R.D., Murase I. Inorg. Chem. 1986, 25, 2160-2163.

15. Kimura E., Machida R., Kodama M. J. Am. Chem. Soc. 1984, 106, 5497-5505.

16. Fujioka H., Kimura E., Kodama M. Chem. Lett. 1982, 737740 .

17. Ikeda I., Katayama T., Okahara M., Shono T. Tetrahedron Lett. 1981, 22, 3615-3616.
18. White B.D., Dishong D.M., Minganti C.,Arnold K.A., Goli D.M., Gokel G.W. Tetrahedron Lett. 1985, 26, 151-154.

19. Zavada J., Koudelka J., Holy P., Belohradsky M., Stibor I. Coll. Czech. Chem. Commun. 1989, 54, 1043-1054.

20. Schultz R.A., White B.D., Dishong D.M., Arnold K.A., Gokel G.W. J. Am. Chem. Soc. 1985, 107, 6659-6668.

21. Dishong D.M., Diamond C.J., Cinoman M.I., Gokel G.W. J. Am. Chem. Soc. 1983, 105, 586-593.

22. Goli D.M., Dishong D.M., Diamond C.J., Gokel G.W. Tetrahedron Lett. 1982, 23, 5243-5246.

23. Nakatsuji Y., Nakamura T., Okahara M., Dishong D.M., Gokel G.W. J. Org. Chem. 1983, 48, 1237-1242.

24. Yakushev A.A., Chernichenko N.M., Anokhin M.V., Averin A.D., Buryak A.K., Denat F., Beletskaya I.P. Molecules 2014, 19, 940-965.

25. Kobelev S.M., Averin A.D., Buryak A.K., Denat F., Guilard R., Beletskaya I.P. Macroheterocycles 2014, 7, 28-33.

26. Anokhin M.V., Averin A.D., Buryak A.K., Beletskaya I.P. Mendeleev Commun. 2011, 21, 132-133.

27. Anokhin M.V., Averin A.D., Beletskaya I.P. Eur. J. Org. Chem. 2011, 6240-6253.

28. Anokhin M.V., Averin A.D., Panchenko S.P., Maloshitskaya O.A., Beletskaya I.P. Russ. J. Org. Chem. 2014, 50, 923-927.

29. Anokhin M.V., Averin A.D., Panchenko S.P., Maloshitskaya O.A., Buryak A.K., Beletskaya I.P. Helv. Chim. Acta 2015, 98, 47-59.

30. Kotovshchikov Yu.N., Latyshev G.V., Lukashev N.V., Beletskaya I.P. Eur. J. Org. Chem. 2013, 7823-7832.

31. Ukai T., Kawazura H., Ishii Y., Bonnet J.J., Ibers J.A. J. Organomet. Chem. 1974, 65, 253-266. 